\title{
N-VALUED REFINED NEUTROSOPHIC SOFT SETS AND THEIR APPLICATIONS IN DECISION MAKING PROBLEMS AND MEDICAL DIAGNOSIS
}

\author{
Shawkat Alkhazaleh ${ }^{1}$, Ayman A. Hazaymeh ${ }^{2}$ \\ ${ }^{1}$ Department of Mathematics \\ Faculty of Science and Art \\ Shaqra University, Saudi Arabia \\ ${ }^{2}$ Department of Mathematics \\ Faculty of Science and Information Technology \\ Jadara University, Jordan
}

Submitted: 3th January 2017; accepted: 22th March 2017

\begin{abstract}
In this work we use the concept of a 'n'-valued refined neutrosophic soft sets and its properties to solve decision making problems, Also a similarity measure between two 'n'valued refined neutrosophic soft sets are proposed. A medical diagnosis (MD) method is established for 'n'-valued refined neutrosophic soft set setting using similarity measures. Lastly a numerical example is given to demonstrate the possible application of similarity measures in medical diagnosis (MD).
\end{abstract}

Keywords: neutrosophic set, neutrosophic soft set, 'n'-valued refined neutrosophic set, 'n'-valued refined neutrosophic soft set, similarity measures, decision making, medical diagnosis.

\section{Introduction}

Neutrosophic set was introduced in 1995 by Florentin Smarandache, who coined the words "neutrosophy" and its derivative "neutrosophic". Then, he introduced the concept of neutrosophic set in [18] and [17] which is a mathematical tool for handling problems involving imprecise, indeterminacy and inconsistent data. Smarandache in 2005 [20] also studied the relation between neutrosophic set and intuitionistic fuzzy sets and he showed that the neutrosophic set is a generalization of the intuitionistic fuzzy sets. Neutrosophy is a new branch of philosophy that studies the origin, nature, and scope of neutralities, as well as their interactions with different ideational spectra. This theory considers every notion or idea $\langle A\rangle$ together with its opposite or negation $\langle$ antiA $\rangle$ and with their spectrum of neutralities $\langle$ neut $A\rangle$ between them (i.e. notions or ideas supporting neither $\langle A\rangle$ nor $\langle$ antiA $\rangle$ ). The neutrosophic numerical components $(t, i, f)$ are crisp numbers, intervals, or in general subsets of the unitary standard or nonstandard unit interval. In 2015 Smarandache [21] presented a short history of logics: from particular cases of 2-symbol or numerical valued logic to the general case of n-symbol or numerical valued logic. He showed generalizations of 2-valued Boolean logic to fuzzy logic, also from the Kleene's and Lukasiewicz' 3symbol valued logics or Belnap's 4-symbol valued logic to the most general n-symbol or numerical valued refined neutrosophic logic. He also gave gave a generalizations for 'n'-valued refined neutrosophic set. In 2015 Agboola [1] developed re- 
fined neutrosophic algebraic structures by studding refined neutrosophic group and presented some of its elementary properties. Broumi et. al. in [6] defined the concept of 'n'-valued interval neutrosophic sets and introduced the basic operations of this concept. Some distances between 'n'-valued interval neutrosophic sets were proposed. They also proposed an efficient approach for group multicriteria decision making based on 'n'-valued interval neutrosophic sets and give an application of 'n'valued interval neutrosophic sets in medical diagnosis problem. Smarandache in 2015 [19] gave a short history about: the neutrosophic set, neutrosophic numerical components and neutrosophic literal components, neutrosophic numbers, neutrosophic intervals, neutrosophic dual number, neutrosophic special dual number, neutrosophic special quasi dual number, neutrosophic linguistic number, neutrosophic linguistic interval-style number, neutrosophic hypercomplex numbers of dimension $n$, and elementary neutrosophic algebraic structures. $\mathrm{He}$ also gave their generalizations to refined neutrosophic set, respectively refined neutrosophic numerical and literal components, then refined neutrosophic numbers and refined neutrosophic algebraic structures, and set-style neutrosophic numbers. A short history of logics: from particular cases of 2-symbol or numerical valued logic to the general case of $n$-symbol or numerical valued logic as presented in [21], [23], [22]. Then some authors studied the similarity measures between some of these concepts such as: Broumi and Smarandache in 2014 [8] proposed the cosine similarity measure of neutrosophic refined (multi-) sets where the cosine similarity measure of neutrosophic refined sets is the extension of improved cosine similarity measure of single valued neutrosophic. They also presented the application of medical diagnosis using this cosine similarity measure of neutrosophic refined set. Also they in 2015 [9] presented a new distance measure between neutrosophic refined sets on the basis of extended Hausdorff distance of neutrosophic set and studied some of their basic properties and using this extended to solve medical diagnosis problem. In 2016 Broumi and Deli [5] proposed the correlation measure of neutrosophic refined (multi-) sets where the concept of this correlation measure of neutrosophic refined sets is the extension of correlation measure of neutrosophic sets and intuitionistic fuzzy multi-sets. They using this measure to solve medical diagnosis and pattern recognition problems. Since the similarity measure is an important tool in pattern recognition and fault diagnosis, Jun Ye in 2015 [11] proposed two cotangent similarity measures for single-valued neutrosophic sets (SVNSs) based on cotangent function. Then, he introduced the weighted cotangent similarity measures and studied the comparison between the cotangent similarity measures of SVNSs and existing cosine similarity measure of SVNSs. He also applied the cotangent similarity measures to the fault diagnosis of steam turbine. In 1999, Molodtsov [15] initiated a novel concept of soft set theory as a new mathematical tool for dealing with uncertainties. Maji et. al. [14] in 2003 studied soft set and gave some operations related to this theory. As a combination of neutrosophic set and soft set, Maji [13] introduced neutrosophic soft set (NSS in short). He established its application in decision making. In 2013 Said and Smarandache [7] defined the concept of intuitionistic neutrosophic soft set and introduced some operations on intuitionistic neutrosophic soft set and some properties of this concept have been established. Mehmet et. al. in 2015 [10] introduced the concept of neutrosophic soft expert set they also defined its basic operations, namely complement, union, intersection, AND and $\mathrm{OR}$, and studied some of their properties and gave an application of this concept in a decision- making problem. Alkhazaleh [3] in 2016 introduced the concept of time-neutrosophic soft set as a generalization of neutrosophic soft set and studied some of its properties. Also, he defined its basic operations, complement, union, intersection, "AND" and "OR" and studied their properties and gave hypothetical application of this concept in decision making problems. In 2015 Karaaslan [12] Studied the concept of single-valued neutrosophic refined soft set as an extension of single-valued neutrosophic refined set. Also, he defined the set of theoretical operations between two single-valued neutrosophic refined soft sets and investigated some basic properties of these operations. He also proposed two methods to calculate correlation coefficient between two single-valued neutrosophic refined soft sets, and gave a clustering analysis application of one of proposed methods. In 2015 Mukherjee and Sarkar [16] proposed a new method of measuring similarity measure and weighted similarity measure between two neutrosophic soft sets (NSSs). They 
also gave a comparative study between the existing similarity measures for neutrosophic soft sets. A decision making method is established for neutrosophic soft set setting using similarity measures and they gave a numerical example to demonstrate the possible application of similarity measures in pattern recognition problems. As a generalization of neutrosophic soft set, Alkhazaleh in 2016 [2] introduced the concept of 'n'-valued refined neutrosophic soft set and studied some of its properties. $\mathrm{He}$ also, defined its basic operations, complement, union intersection, "AND" and "OR" and studied their properties. In this study, after giving some definitions related to the ' $n$ '-valued refined neutrosophic soft set (n-VRNS-set), we use these concepts to solve decision making problems. Also, a similarity measure between two 'n'-valued refined neutrosophic soft sets are proposed. A medical diagnosis method (MD) is established for 'n'-valued refined neutrosophic soft set setting using similarity measures. Lastly, a numerical example is given to demonstrate the possible application of similarity measures in medical diagnosis (MD).

\section{Preliminary}

In this Section we recall some definitions and properties regarding neutrosophic set theory, soft set theory, 'n'-valued refined neutrosophic, neutrosophic soft set theory and 'n'-valued refined neutrosophic soft set required in this paper.

Definition 1 [20] A neutrosophic set A on the universe of discourse $X$ is defined as

$$
A=\left\{<x ; T_{A}(x) ; I_{A}(x) ; F_{A}(x)>; x \in X\right\}
$$

where $T ; I ; F: X \rightarrow]^{-} 0 ; 1^{+}[$and

$$
{ }^{-} 0 \leqslant T_{A}(x)+I_{A}(x)+F_{A}(x) \leq 3^{+} .
$$

Molodtsov defined soft set in the following way. Let $U$ be a universe and $E$ be a set of parameters. Let $P(U)$ denote the power set of $U$ and $A \subseteq E$.

Definition 2 [15] A pair $(F, A)$ is called a soft set over $U$, where $F$ is a mapping

$$
F: A \rightarrow P(U) .
$$

In other words, a soft set over $U$ is a parameterized family of subsets of the universe $U$. For $\varepsilon \in A, F(\varepsilon)$ may be considered as the set of $\varepsilon$-approximate elements of the soft set $(F, A)$.

Definition 3 [13] Let $U$ be an initial universe set and $E$ be a set of parameters. Consider $A \subset E$. Let $P(U)$ denotes the set of all neutrosophic sets of $U$. The collection $(F, A)$ is termed to be the soft neutrosophic set over $U$, where $F$ is a mapping given by $F: A \rightarrow P(U)$.

Definition 4 [2] Let $U$ be an initial universe set and $E$ be a set of parameters, $T_{j}=\left\{T_{1}, T_{2}, \ldots, T_{p}\right\}$ be a set types of truths, $I_{k}=\left\{I_{1}, I_{2}, \ldots, I_{r}\right\}$ be a set types of indeterminacies and $F_{k}=\left\{F_{1}, F_{2}, \ldots, F_{s}\right\}$ be a set types of falsities and $n=p+r+s$ where all subcomponents $T_{j} ; I_{k} ; F_{l}$ subsets of $[0,1]$. Consider $A \subset E$. Let $P(U)$ denotes the set of all ' $n$ '-valued refined neutrosophic sets of $U$. The collection $\left(f_{n}, A\right)$ is termed to be the 'n'-valued refined neutrosophic soft set over $U$, where $f_{n}$ is a mapping given by $f_{n}: A \rightarrow P(U)$.

\section{An Application of n-VRNSS in Decision Making}

In this Section, we present an application of nVRNSS in a decision making problem.

Assume that a company wants to fill a position. There are two candidates who form the universe $U=\left\{u_{1}, u_{2}\right\}$, the hiring committee considers a set of parameters, $E=\left\{e_{1}, e_{2}, e_{3}\right\}$, the parameters $e_{i}(i=1,2,3)$ stand for "experience", "computer knowledge" and "good speaking", respectively. Let the truth $T$ is refined (split) as $T_{A}=$ AbsoluteTruth, $T_{R}=$ RelativeTruth, indeterminacy $I$ is refined (split) as $I_{A}=$ AbsoluteIndeterminacy, $I_{R}=$ RelativeIndeterminacy and and the falsity $F$ is refined (split) as $F_{A}=$ Absolutefalsity, $I_{R}=$ Relativefalsity . $T_{A}, T_{R}, I_{A}, I_{R} F_{A}$ and $F_{R}$ are subsets of $[0,1]$, such that $n=p+r+s$ where $n=6, p=2, r=2, s=2$. Then, after a serious discussion the committee constructs the following case of 6-Valued refined neutrosophic soft set. 


\section{Example 1 [2]}

$$
\begin{aligned}
& \left\{\frac{u_{1}\left(e_{1}\right)=}{\langle(0.5,0.4) ;(0.2,0.3) ;(0.4,0.3)\rangle}, \frac{u_{2}}{\langle(0.7,0.4) ;(0.1,0.5) ;(0.5,0.1)\rangle}\right\}, \\
& \left\{\frac{u_{1} f_{5}\left(e_{2}\right)=}{\langle(0.3,0.5) ;(0.3,0.4) ;(0.5,0.4)\rangle}, \frac{u_{2}}{\langle(0.3,0.3) ;(0.2,0.3) ;(0.4,0.8)\rangle}\right\}, \\
& \left\{\frac{u_{1} f_{5}\left(e_{3}\right)=}{\langle(0.6,0.3) ;(0.3,0.1) ;(0.2,0.4)\rangle}, \frac{u_{2}}{\langle(0.5,0.7) ;(0.1,0.2) ;(0.2,0.2)\rangle}\right\},
\end{aligned}
$$

and we can write the 6-valued refined neutrosophic soft set $\left(f_{6}, E\right)$ as consisting of the following collection of approximations:

$$
\begin{aligned}
& \left(f_{6}, E\right)= \\
& \left\{\left(e_{1},\left\{\frac{u_{1}}{\langle(0.5,0.4) ;(0.2,0.3) ;(0.4,0.3)\rangle}, \frac{u_{2}}{\langle(0.7,0.4) ;(0.1,0.5) ;(0.5,0.1)\rangle}\right\}\right),\right. \\
& \left(e_{2},\left\{\frac{u_{1}}{\langle(0.3,0.5) ;(0.3,0.4) ;(0.5,0.4)\rangle}, \frac{u_{2}}{\langle(0.3,0.3) ;(0.2,0.3) ;(0.4,0.8)\rangle}\right\}\right) \text {, } \\
& \left.\left(e_{3},\left\{\frac{u_{1}}{\langle(0.6,0.3) ;(0.3,0.1) ;(0.2,0.4)\rangle}, \frac{u_{2}}{\langle(0.5,0.7) ;(0.1,0.2) ;(0.2,0.2)\rangle}\right\}\right)\right\} .
\end{aligned}
$$

Also we can represent the above set as shown in Table 1 .

The following algorithm may be followed by the company to fill the position.

1 Input the n-VRNSS $\left(F_{n}, E\right)$.

2 Find the neutrosophic soft set $(F, E)$,(Table 2) where:

a $\forall e \in E$, the truth-memberships $T_{\left(u_{i}, e\right)}=$ $\frac{1}{p} \sum_{j=1}^{p} T_{j}\left(u_{i}, e\right)$.

$\mathrm{b} \forall e \in E$, the indeterminacy-memberships $I_{\left(u_{i}, e\right)}=\frac{1}{r} \sum_{j=1}^{r} I_{j}\left(u_{i}, e\right)$.

c $\forall e \in E$, the falsity-memberships $F_{\left(u_{i}, e\right)}=$ $\frac{1}{s} \sum_{j=1}^{s} F_{j}\left(u_{i}, e_{i}\right)$.
3 Find $\alpha_{j}=\sum_{i} T\left(u_{i j}\right)$.

4 Find $\beta_{j}=\sum_{i} I\left(u_{i j}\right)$.

5 Find $\gamma_{j}=\sum_{i} F\left(u_{i j}\right)$.

6 Find $\delta_{j}=\alpha_{j}+\beta_{j}-\gamma_{j}$.

7 Find $m$, for which $\delta_{m}=\max \delta_{j}$. Then $\mathrm{s}_{m}$ is the optimal choice object. If $m$ has more than one value, then any one of them could be chosen by the company using its option.

Now we use this algorithm to find the best choice for the company to fill the position. From Table 2 we have the following: $\max \delta_{j}=\delta_{2}$, so the committee will choose candidate 2 for the job. 
Table 1. 6-valued refined neutrosophic soft set $\left(f_{6}, E\right)$

\begin{tabular}{|c|c|c|c|c|c|c|}
\hline & $\left(u_{1}, e_{1}\right)$ & $\left(u_{1}, e_{2}\right)$ & $\left(u_{1}, e_{3}\right)$ & $\left(u_{2}, e_{1}\right)$ & $\left(u_{2}, e_{2}\right)$ & $\left(u_{2}, e_{3}\right)$ \\
\hline$T_{A}$ & 0.5 & 0.3 & 0.6 & 0.7 & 0.3 & 0.5 \\
\hline$T_{R}$ & 0.4 & 0.5 & 0.3 & 0.4 & 0.3 & 0.7 \\
\hline$I_{A}$ & 0.2 & 0.3 & 0.3 & 0.1 & 0.2 & 0.1 \\
\hline$I_{R}$ & 0.3 & 0.4 & 0.1 & 0.5 & 0.3 & 0.2 \\
\hline$F_{A}$ & 0.4 & 0.5 & 0.2 & 0.5 & 0.4 & 0.2 \\
\hline$F_{R}$ & 0.3 & 0.4 & 0.4 & 0.1 & 0.8 & 0.2 \\
\hline
\end{tabular}

Table 2. Neutrosophic soft set $(f, E)$

\begin{tabular}{|c|c|c|c|c|c|c|}
\hline & $\left(u_{1}, e_{1}\right)$ & $\left(u_{1}, e_{2}\right)$ & $\left(u_{1}, e_{3}\right)$ & $\left(u_{2}, e_{1}\right)$ & $\left(u_{2}, e_{2}\right)$ & $\left(u_{2}, e_{3}\right)$ \\
\hline$T$ & 0.45 & 0.4 & 0.45 & 0.55 & 0.3 & 0.6 \\
\hline$I$ & 0.25 & 0.35 & 0.2 & 0.3 & 0.25 & 0.15 \\
\hline$F$ & 0.35 & 0.45 & 0.3 & 0.3 & 0.6 & 0.2 \\
\hline
\end{tabular}

\section{Similarity Measure for 'n'- valued Refined Neutrosophic Soft Set(n-VRNSSs)}

In this Section we have proposed a new method for measuring similarity measure and weighted similarity measure for $\mathrm{n}$-VRNSSs and some basic properties are also studied.

Definition 5 Let $U=\left\{x_{1}, x_{2}, x_{3}, \ldots, x_{d}\right\}$ be the universe of discourse and $E=\left\{e_{1}, e_{2}, e_{3}, \ldots, e_{k}\right\}$ be the set of parameters and let $\left(f_{n}, E\right)$ and $\left(g_{n}, E\right)$ be two ' $n$ '-valued refined neutrosophic soft sets over $U(E)$. Then the similarity measure between $n$ $\operatorname{VNSSS}\left(f_{n}, E\right)$ and $\left(g_{n}, E\right)$ where $n=p+r+s$, is denoted by $S\left(f_{n}, g_{n}\right)$ and is defined as follows

$$
\begin{array}{r}
S\left(f_{n}, g_{n}\right)=\frac{1}{3 d k} \sum_{i=1}^{d} \sum_{j=1}^{k}\left(3-\frac{1}{p} \sum_{l=1}^{p}\left|T^{l} f_{n}\left(x_{i}\right)\left(e_{j}\right)-T^{l}{ }_{g_{n}}\left(x_{i}\right)\left(e_{j}\right)\right|\right. \\
-\frac{1}{r} \sum_{l=1}^{r}\left|I^{l} f_{n}\left(x_{i}\right)\left(e_{j}\right)-I^{l} g_{n}\left(x_{i}\right)\left(e_{j}\right)\right| \\
\left.-\frac{1}{S} \sum_{l=1}^{s}\left|F^{l} f_{n}\left(x_{i}\right)\left(e_{j}\right)-F^{l}{ }_{g_{n}}\left(x_{i}\right)\left(e_{j}\right)\right|\right)
\end{array}
$$

Example 2 Let $U=\left\{u_{1}, u_{2}\right\}$ be a set of universe, $E=\left\{e_{1}, e_{2}, e_{3}\right\}$ a set of parameters. Let the Indeterminacy I is refined (split) as Un $=U n k n o w n$, and $C=$ contradiction. $T, F, U n$ and $C$ are subsets of $[0,1]$. Let:

$\left\{\left(e_{1},\left\{\frac{u_{1}}{\langle 0.3 ;(0.3,0.5) ; 0.2\rangle}, \frac{u_{2}}{\langle 0.8 ;(0.3,0.6) ; 0.3\rangle}\right\}\right)\right.$, $\left(e_{2},\left\{\frac{u_{1}}{\langle 0.4 ;(0.4,0.6) ; 0.4\rangle}, \frac{u_{2}}{\langle 0.5 ;(0.3,0.4) ; 0.3\rangle}\right\}\right)$,

$\left.\left(e_{3},\left\{\frac{u_{1}}{\langle 0.7 ;(0.5,0.1) ; 0.3\rangle}, \frac{u_{2}}{\langle 0.6 ;(0.3,0.1) ; 0.3\rangle}\right\}\right)\right\}$.

and

$\left\{\left(e_{1},\left\{\frac{u_{1}}{\langle 0.5 ;(0.2,0.3) ; 0.4\rangle}, \frac{u_{2}}{\langle 0.7 ;(0.1,0.5) ; 0.5\rangle}\right\}\right)\right.$,

$\left(e_{2},\left\{\frac{u_{1}}{\langle 0.3 ;(0.3,0.4) ; 0.5\rangle}, \frac{u_{2}}{\langle 0.3 ;(0.2,0.3) ; 0.4\rangle}\right\}\right)$,

$\left.\left(e_{3},\left\{\frac{u_{1}}{\langle 0.6 ;(0.3,0.1) ; 0.2\rangle}, \frac{u_{2}}{\langle 0.5 ;(0.1,0.2) ; 0.2\rangle}\right\}\right)\right\}$.

Then we can represent the above sets as shown in Table 6 and 5 .

Now by using Definition 5 we can found the similarity measure between $\left(f_{4}, E\right)$ and $\left(g_{4}, E\right)$ as follows

$$
\begin{array}{r}
S\left(f_{4}, g_{4}\right)=\frac{1}{18} \sum_{i=1}^{2} \sum_{j=1}^{3}\left(3-\frac{1}{1} \sum_{l=1}^{1}\left|T^{l} f_{f}\left(u_{i}\right)\left(e_{j}\right)-T^{l}{ }_{g}\left(u_{i}\right)\left(e_{j}\right)\right|\right. \\
-\frac{1}{2} \sum_{l=1}^{2}\left|I^{l}{ }_{f}\left(u_{i}\right)\left(e_{j}\right)-I^{l}{ }_{g}^{l}\left(u_{i}\right)\left(e_{j}\right)\right| \\
\left.-\frac{1}{1} \sum_{l=1}^{1}\left|F^{l}{ }_{f}\left(u_{i}\right)\left(e_{j}\right)-F^{l}{ }_{g}\left(u_{i}\right)\left(e_{j}\right)\right|\right) \\
=\frac{1}{18} \sum_{i=1}^{2} \sum_{j=1}^{3}\left(3-\left|T_{f}\left(u_{i}\right)\left(e_{j}\right)-T_{g}\left(u_{i}\right)\left(e_{j}\right)\right|\right. \\
-\frac{1}{2} \sum_{l=1}^{2}\left|I^{l}{ }_{f}\left(u_{i}\right)\left(e_{j}\right)-I_{g}^{l}\left(u_{i}\right)\left(e_{j}\right)\right| \\
\left.-\left|F_{f}\left(u_{i}\right)\left(e_{j}\right)-F_{g}\left(u_{i}\right)\left(e_{j}\right)\right|\right)
\end{array}
$$


Table 3. $\delta_{j}=\alpha_{j}+\beta_{j}-\gamma_{j}$.

\begin{tabular}{|c|c|c|c|}
\hline$\alpha_{j}=\sum_{i} u_{i j}$ & $\beta_{j}=\sum_{i} u_{i j}$ & $\gamma_{j}=\sum_{i} u_{i j}$ & $\delta_{j}=\alpha_{j}+\beta_{j}-\gamma_{j}$. \\
\hline$\alpha_{1}=1.3$ & $\beta_{1}=0.8$ & $\gamma_{1}=1.1$ & $\delta_{1}=1$ \\
\hline$\alpha_{2}=1.45$ & $\beta_{2}=0.7$ & $\gamma_{2}=1.1$ & $\delta_{2}=1.05$ \\
\hline
\end{tabular}

Table 4. 4-valued refined neutrosophic soft set $\left(f_{4}, E\right)$

\begin{tabular}{|c|c|c|c|c|c|c|}
\hline & $\left(u_{1}, e_{1}\right)$ & $\left(u_{1}, e_{2}\right)$ & $\left(u_{1}, e_{3}\right)$ & $\left(u_{2}, e_{1}\right)$ & $\left(u_{2}, e_{2}\right)$ & $\left(u_{2}, e_{3}\right)$ \\
\hline$T$ & 0.3 & 0.4 & 0.7 & 0.8 & 0.5 & 0.6 \\
\hline$U n$ & 0.3 & 0.4 & 0.5 & 0.3 & 0.3 & 0.3 \\
\hline$C$ & 0.5 & 0.6 & 0.1 & 0.6 & 0.4 & 0.1 \\
\hline$F$ & 0.2 & 0.4 & 0.3 & 0.3 & 0.3 & 0.3 \\
\hline
\end{tabular}

After some calculations, the similarity measure between $\left(f_{4}, E\right)$ and $\left(g_{4}, E\right)$ is given by $S\left(f_{4}, g_{4}\right) \simeq$ 0.87 .

Theorem 1 If $S\left(f_{n}, g_{n}\right)$ be the similarity measure between two $n$-VRNSSs $\left(f_{n}, E\right)$ and $\left(g_{n}, E\right)$ then:

$$
\begin{aligned}
& \text { 1. } 0 \leq S\left(f_{n}, g_{n}\right) \leq 1, \\
& \text { 2. } S\left(f_{n}, g_{n}\right)=S\left(g_{n}, f_{n}\right), \\
& \text { 3. } S\left(f_{n}, f_{n}\right)=1, \\
& \text { 4. }\left(f_{n}, E\right) \cap\left(g_{n}, E\right)=\phi \Leftrightarrow S\left(f_{n}, g_{n}\right)=0 . \\
& \text { 5. If }\left(f_{n}, E\right) \subseteq\left(g_{n}, E\right) \text { and }\left(g_{n}, E\right) \subseteq \\
& \quad\left(z_{n}, E\right) \text {, then } S\left(f_{n}, z_{n}\right) \leq S\left(g_{n}, z_{n}\right) .
\end{aligned}
$$

Proof The proof is straightforward from Definition 5.

Definition 6 Let $\left(f_{n}, E\right)$ and $\left(g_{n}, E\right)$ be two $n$ VRNSSS over the same universe $(U ; E)$. We call the two $n$-VRNSSs to be significantly similar if $S\left(f_{n}, g_{n}\right) \geq \frac{1}{2}$

\section{Application of the Similarity Measure in Medical Diagnosis}

In the following example, given by Alkhazaleh and Salleh [4] we will try to estimate the possibility that a sick person having certain visible symptoms is suffering from dengue fever. For this we first construct a model n-VRNSS for dengue fever and the n-VRNSS of symptoms for the sick person. Next we find the similarity measure of these two sets.
If they are significantly similar then we conclude that the person is possibly suffering from dengue fever. Let the Indeterminacy $I$ is refined (split) as $U n=$ Unknown, and $C=$ contradiction. $T, F, U n$ and $C$ are subsets of $[0,1]$. Let our universal set contain only two elements "yes" and "no", that is, $U=\{y, n\}$. Here the set of parameters $E$ is the set of certain visible symptoms. Let $E=\left\{e_{1}, e_{2}, e_{3}, e_{4}, e_{5}, e_{6}, e_{7}, e_{8}, e_{9}, e_{10}, e_{11}\right\}$, where $e_{1}=$ body temperature, $e_{2}=$ cough with chest congestion, $e_{3}=$ loose motion, $e_{4}=$ chills, $e_{5}=$ headache, $e_{6}=$ low heart rate (bradycardia), $e_{7}=$ pain upon moving the eyes, $e_{8}=$ breathing trouble, $e_{9}=$ a flushing or pale pink rash comes over the face, $e_{10}=$ low blood pressure (hypotension) and $e_{11}=$ loss of appetite.

Our model n-VRNSS for dengue fever $f_{4}$ is given in Table 6 and this can be prepared with the help of a physician. After talking to the sick person we can construct his n-VRNSS $g_{4}$ as in Table 6. Now we find the similarity measure of these two sets (as in Example 2), here $S\left(f_{4}, g_{4}\right) \cong 0.62<\frac{1}{2}$. Hence the two n-VRNSSs are significantly similar. Therefore, we conclude that the person is suffering from dengue fever.

\section{Acknowledgments}

The authors would like to acknowledge the financial support received from Shaqra University. 
Table 5. 4-valued refined neutrosophic soft set $\left(g_{4}, E\right)$

\begin{tabular}{|c|c|c|c|c|c|c|}
\hline & $\left(u_{1}, e_{1}\right)$ & $\left(u_{1}, e_{2}\right)$ & $\left(u_{1}, e_{3}\right)$ & $\left(u_{2}, e_{1}\right)$ & $\left(u_{2}, e_{2}\right)$ & $\left(u_{2}, e_{3}\right)$ \\
\hline$T$ & 0.5 & 0.3 & 0.6 & 0.7 & 0.3 & 0.5 \\
\hline$U n$ & 0.2 & 0.3 & 0.3 & 0.1 & 0.2 & 0.1 \\
\hline$C$ & 0.3 & 0.4 & 0.1 & 0.5 & 0.3 & 0.2 \\
\hline$F$ & 0.4 & 0.5 & 0.2 & 0.5 & 0.4 & 0.2 \\
\hline
\end{tabular}

Table 6. Model n-VRNSS for dengue fever and sick person

\begin{tabular}{|c|c|c|c|c|}
\hline & \multicolumn{2}{|c|}{ Model n-VRNSS for dengue fever $f_{4}$} & \multicolumn{2}{c|}{ Model n-VRNSS for sick person $g_{4}$} \\
\hline$e_{i}$ & $y$ & $n$ & $y$ & $n$ \\
\hline$e_{1}$ & $\langle 1 ;(0,0) ; 0\rangle$ & $\langle 0 ;(0,0) ; 1\rangle$ & $\langle 0.4 ;(0.2,0.3) ; 0.5\rangle$ & $\langle 0.8 ;(0.2,0.2) ; 0.2\rangle$ \\
\hline$e_{2}$ & $\langle 0 ;(0,0) ; 1\rangle$ & $\langle 1 ;(0,0) ; 0\rangle$ & $\langle 0.8 ;(0.3,0.2) ; 0.1\rangle$ & $\langle 0.3 ;(0.1,0.2) ; 0.7\rangle$ \\
\hline$e_{3}$ & $\langle 0 ;(0,0) ; 1\rangle$ & $\langle 1 ;(0,0) ; 0\rangle$ & $\langle 0.5 ;(0.4,0.2) ; 0.4\rangle$ & $\langle 0.4 ;(0.2,0.4) ; 0.5\rangle$ \\
\hline$e_{4}$ & $\langle 1 ;(0,0) ; 0\rangle$ & $\langle 0 ;(0,0) ; 1\rangle$ & $\langle 0.7 ;(0.4,0.2) ; 0.4\rangle$ & $\langle 0.3 ;(0.3,0.2) ; 0.5\rangle$ \\
\hline$e_{5}$ & $\langle 1 ;(0,0) ; 0\rangle$ & $\langle 0 ;(0,0) ; 1\rangle$ & $\langle 0.9 ;(0.3,0.4) ; 0.2\rangle$ & $\langle 0.1 ;(0.2,0.4) ; 0.8\rangle$ \\
\hline$e_{6}$ & $\langle 1 ;(0,0) ; 0\rangle$ & $\langle 0 ;(0,0) ; 1\rangle$ & $\langle 0.6 ;(0.5,0.3) ; 0.3\rangle$ & $\langle 0.2 ;(0.3,0.2) ; 0.6\rangle$ \\
\hline$e_{7}$ & $\langle 1 ;(0,0) ; 0\rangle$ & $\langle 0 ;(0,0) ; 1\rangle$ & $\langle 0.5 ;(0.4,0.1) ; 0.3\rangle$ & $\langle 0.3 ;(0.3,0.3) ; 0.6\rangle$ \\
\hline$e_{8}$ & $\langle 0 ;(0,0) ; 1\rangle$ & $\langle 1 ;(0,0) ; 0\rangle$ & $\langle 0.8 ;(0.3,0.3) ; 0.5\rangle$ & $\langle 0.2 ;(0.1,0.4) ; 0.6\rangle$ \\
\hline$e_{9}$ & $\langle 1 ;(0,0) ; 0\rangle$ & $\langle 0 ;(0,0) ; 1\rangle$ & $\langle 0.7 ;(0.2,0.1) ; 0.4\rangle$ & $\langle 0.3 ;(0.1,0.1) ; 0.5\rangle$ \\
\hline$e_{10}$ & $\langle 1 ;(0,0) ; 0\rangle$ & $\langle 0 ;(0,0) ; 1\rangle$ & $\langle 0.6 ;(0.1,0.1) ; 0.3\rangle$ & $\langle 0.4 ;(0.2,0.3) ; 0.5\rangle$ \\
\hline$e_{11}$ & $\langle 0 ;(0,0) ; 1\rangle$ & $\langle 1 ;(0,0) ; 0\rangle$ & $\langle 0.3 ;(0.2,0.2) ; 0.6\rangle$ & $\langle 0.6 ;(0.1,0.4) ; 0.3\rangle$ \\
\hline
\end{tabular}

\section{References}

[1] A. A. A. Agboola, On refined neutrosophic algebraic structures, Neutrosophic Sets and Systems, 10:99-101, 2015.

[2] S. Alkhazaleh, 'n'-valued refined neutrosophic soft set theory, Journal of Intelligent \& Fuzzy Systems 32 (6), pp. 4311-4318.

[3] S. Alkhazaleh, Time-neutrosophic soft set and its applications, Journal of Intelligent \& Fuzzy Systems, 30(2):1087-1098, 2016.

[4] S. Alkhazaleh and A. R. Salleh, Possibility fuzzy soft set, Advances in Decision Sciences, page 18, 2011.

[5] S. Broumi and I. Deli, Correlation measure for neutrosophic refined sets and its application in medical diagnosis, Palestine Journal of Mathematics, 5(1):135-143, 2016.

[6] S. Broumi, I. Deli, and F. Smarandache, Critical Review, volume X, chapter 'n'-valued Interval Neutrosophic Sets and Their Application in Medical Diagnosis, pages 45-69, Center for Mathematics of Uncertainty, Creighton University, USA, 2015.
[7] S. Broumi and F. Smarandache, Intuitionistic neutrosophic soft set, Journal of Information and Computing Science, 8(2):130-140, 2013.

[8] S. Broumi and F. Smarandache, Neutrosophic refined similarity measure based on cosine function, Neutrosophic Sets and Systems, 6:42-48, 2014.

[9] S. Broumi and F. Smarandache, Extended hausdorff distance and similarity measures for neutrosophic refined sets and their application in medical diagnosis, Journal of New Theory, 7:64-78, 2015.

[10] M. Şahin, S. Alkhazaleh, and V. Uluçay, Neutrosophic soft expert sets, Applied Mathematics, 6:116-127, 2015.

[11] Y. Jun, Single-valued neutrosophic similarity measures based on cotangent function and their application in the fault diagnosis of steam turbine, Soft Computing, pages 1-9, 2015.

[12] F. Karaaslan, Correlation coefficients of singlevalued neutrosophic refined soft sets and their applications in clustering analysis, Neural Computing and Applications, 2016:1-13, 2016.

[13] P. Maji, Neutrosophic soft set, Annals of Fuzzy Mathematics and Informatics, 5(1):157-168, 2013. 
[14] P. Maji, A. Roy, and R. Biswas, Soft set theory, Computers and Mathematics with Applications, 45:555-562, 2003.

[15] D. Molodtsov, Soft set theory-first results, Computers and Mathematics with Applications, 37:1931, 1999.

[16] A. Mukherjee and S. Sarkar, A new method of measuring similarity between two neutrosophic soft sets and its application in pattern recognition problems, Neutrosophic Sets and Systems, 8:63-68, 2015.

[17] F. Smarandache, Neutrosophic logic and set, mss, 1995.

[18] F. Smarandache, Neutrosophy, Neutrosophic Probability, Set, and Logic, Amer. Res. Press, Rehoboth, USA, 1998.

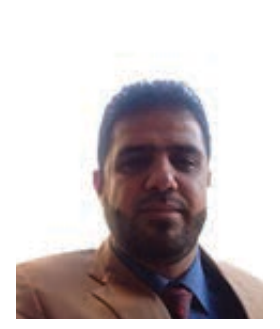

Shawkat Alkhazaleh: Associate Professor, Mathematics Department, Faculty of Science and Arts, Shaqra University, Sajer Branch. He interested in the field of soft sets, fuzzy set, neutrosophic set and there application in decision-making and medical diagnosis problem. He has many papers in this field. He received his PhD in 2012 from the National University of Malaysia UKM.
[19] F. Smarandache, A Unifying Field in Logics, Neutrosophy: Neutrosophic Probability, Set and Logic, Amer. Res. Press, Rehoboth, USA, 1999.

[20] F. Smarandache, Neutrosophic set, a generalization of the intuitionistic fuzzy sets, International Journal of Pure and Applied Mathematics, 24:287-297, 20055.

[21] F. Smarandache, 'n'-valued refined neutrosophic logic and its applications in physics, Progress in Physics, 4:143-146, 2013.

[22] F. Smarandache, Refined literal indeterminacy and the multiplication law of subindeterminacies, Neutrosophic Sets and Systems, 9:1-7, 2015.

[23] F. Smarandache, (t,i,f)-neutrosophic structures and i neutrosophic structures, Neutrosophic Sets and Systems, 8:3-10, 2015.

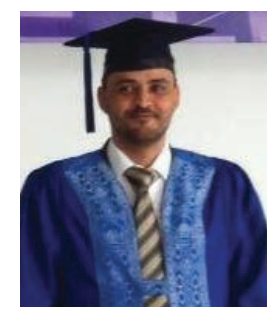

Ayman A. Hazaymeh: Assistant Professor, Mathematics Department, Faculty of Science and Information Technology, Jadara University, He interested in the field of soft sets, fuzzy set, neutrosophic set and there application in decision-making and medical diagnosis problem. He has many papers in this field. He received his $\mathrm{PhD}$ in 2013 from the Islamic Science University of Malaysia (USIM). 